\title{
A 10-Year Retrospective Clinical Analysis of Fungal Keratitis in a Portuguese Tertiary Centre
}

This article was published in the following Dove Press journal:

Clinical Ophthalmology

\author{
Ana Maria Cunha (1D) \\ João Tiago Loja $\mathbb{D}^{2}$ \\ Luís Torrão (iD) \\ Raúl Moreira' \\ Dolores Pinheiro (iD ${ }^{3}$ \\ Fernando Falcão-Reis ${ }^{1,4}$ \\ João Pinheiro-Costa (iD) ${ }^{1,5}$ \\ 'Department of Ophthalmology, Centro \\ Hospitalar Universitário São João, Porto, \\ Portugal; ${ }^{2}$ Faculty of Medicine, University \\ of Porto, Porto, Portugal; ${ }^{3}$ Laboratory of \\ Microbiology, Service of Clinical \\ Pathology, Centro Hospitalar \\ Universitário São João, Porto, Portugal; \\ ${ }^{4}$ Department of Surgery and Physiology, \\ Faculty of Medicine, University of Porto, \\ Porto, Portugal; ${ }^{5}$ Department of \\ Biomedicine, Faculty of Medicine, \\ University of Porto, Porto, Portugal
}

Correspondence: Ana Maria Cunha Department of Ophthalmology of São João Hospital, Centro Hospitalar de São João,

Avenida Prof. Hernâni Monteiro, Porto

$4202-45$ I, Portugal

Tel +35 I 2255I 2100

Fax +35I 2255I3669

Email ana.cunha.18@gmail.com
Purpose: To determine the microbiological profile, risk factors, treatment and surgical intervention rates of fungal keratitis at a tertiary referral centre.

Methods: A retrospective review of microbiological and medical records from hospitalised patients treated for fungal keratitis at Centro Hospitalar Universitário de São João from 2009 to 2019 was conducted.

Results: Overall, 43 patients were included in our study. The mean age of patients was 63.7 years and $46.5 \%$ were men. In culture were isolated $22(51.2 \%)$ filamentous fungi and 21 $(48.8 \%)$ yeast. Candida species $(\mathrm{n}=20,46.5 \%)$, Fusarium species $(\mathrm{n}=10,23.4 \%)$ and Aspergillus species $(\mathrm{n}=4,9.3 \%)$ were the most common isolated species. Important risk factors were contact lens use $(\mathrm{n}=24,55.8 \%)$, long-term users of topical corticosteroids $(\mathrm{n}=$ $19,44.2 \%)$ and previous keratitis $(n=19,44.2 \%)$. Yeast isolates had a statistically significant higher prevalence in long-term users of topical corticosteroids compared to filamentous ones $(\mathrm{p}=0.043)$. Twenty-four cases $(55.8 \%)$ required surgical intervention, of which 23 cases underwent therapeutic penetrating keratoplasty. Ocular complications, such as evisceration was noted in 12 patients $(27.9 \%)$ and endophthalmitis in $5(11.6 \%)$. No statistically significant changes of best corrected visual acuity (BCVA) were found after treatment $(\mathrm{p}=0.687)$.

Conclusion: Most patients with fungal keratitis have associated risk factors. Filamentous and yeast species have equally prevalent etiologies. In general, our results mirror how difficult and challenging the approach and treatment of fungal keratitis could be.

Keywords: fungal keratitis, risk factors, medical therapy, penetrating keratoplasty, visual outcomes

\section{Introduction}

Keratomycosis, better known as fungal keratitis (FK), is an ocular pathology that can severely affect the integrity of the eye. ${ }^{1}$ Indeed, this infection can opacify the cornea and originate vision loss, ${ }^{1}$ being an important cause of blindness in the developing world. ${ }^{2}$ Epidemiologically, FK represents almost $50 \%$ of infectious keratitis in tropical and subtropical areas, with the highest annual incidences of keratomycosis located in South India and Nepal. ${ }^{3}$ In Europe, fungal keratitis is more unusual, especially in its temperate regions. ${ }^{4}$ However, recent data claim an increasing number of keratitis cases in this continent, as it has been recently documented in the United Kingdom. ${ }^{4}$ Filamentous fungi, such as Fusarium and Aspergillus, and yeast like Candida, are the genders more associated with keratitis. ${ }^{1}$

Several predisposing factors could be involved in fungal keratitis, likely corneal trauma, contact lens wear, long term use of topical corticosteroids, penetrating 
keratoplasty (PK), pre-existing herpes simplex virus (HSV) keratitis and diabetes mellitus. ${ }^{3,5}$ Although the pathogenesis of fungal keratitis is not clear, it is believed that a predisposing element for keratitis, such as ocular trauma is responsible for a break in the homeostasis of the ocular surface, in which corneal epithelium is the barrier against infection. ${ }^{1}$ Therefore, the microorganisms can penetrate the cornea's layers with this invasion resulting in an innate and adaptive immune mediated inflammation. ${ }^{5}$

Accurate identification of the aetiological agent of fungal keratitis is of great importance in order to administer appropriate treatment. Conventional culture methods allows causative agent identification and eventual antifungal susceptibility testing, but are time consuming ${ }^{6}$ and, nowadays, polymerase chain reaction (PCR) is assumed as an ideal diagnostic tool, however it should be used cautiously, as commensal agents can also be amplified. ${ }^{3}$

The main drugs for the treatment of fungal disease can be divided into four groups, namely, polyenes, azoles, pyrimidines and echinocandins. Corneal intrastromal injections and intraocular injections into the anterior chamber of agents such as voriconazole and PK could be considered in the surgical treatment. ${ }^{7}$ PK has been documented to be an effective modality for refractory or severe cases of fungal keratitis and a value weapon to treat fungal perforations. ${ }^{1}$ The ideal moment when penetrating keratoplasty should be performed is controversial, although several studies mention better outcomes in patients submitted to keratoplasty early, particularly before perforation or limbal/scleral extension is present. ${ }^{5}$ It is commonly performed after 4 weeks on average of clinical presentation to avoid further extension of the infection. ${ }^{1}$

In this study, we aim to determine the microbiological profile, risk factors, treatment and surgical intervention rates of fungal keratitis at a Portuguese tertiary referral hospital.

\section{Materials and Methods}

A retrospective study of microbiologically documented cases of FK from January 2009 to December 2019 and respective medical records was made at the Ophthalmology Corneal Department of Centro Hospitalar Universitário de São João. It was conducted according to the Helsinki Declaration and was approved by the Ethics Committee of Centro Hospitalar Universitário de São João. Patient's informed consent was not required since it is a retrospective study and patients' names, addresses, and other confidential information were not collected nor shared throughout the study.
In suspected cases of FK, two corneal swabs, from the bottom of the ulcer and from the inferior conjunctival fornix were collected and inoculated in the transport medium. In the microbiology laboratory, the swabs were cultured in blood agar, chocolate agar and Sabouraud medium for the growth of bacteria and fungi, respectively. The first two media were incubated in atmospheric $\mathrm{CO} 2$ at $35^{\circ} \mathrm{C}$ for five days, and the Sabouraud medium was incubated in aerobic conditions at $25^{\circ} \mathrm{C}$ for 15 days.

Demographic features and time from onset of symptoms to hospital admission were evaluated. The following risk factors were also recorded: trauma with vegetable matter, trauma with other object or material, long term use of topical corticosteroids, previous PK, pre-existing HSV keratitis or others previous keratitis, other ocular surface diseases, contact lenses wear, comorbidities (including metabolic syndrome, diabetes mellitus, autoimmune diseases, cardiac pathologies). Additional data recorded included best corrected visual acuity (BCVA) at presentation and at the end of follow-up and prescribed therapy. Patients submitted to PK were followed for at least 6 months. Analysed outcomes were the need for therapeutic PK, BCVA at the end of follow-up and loss of eyeball integrity. BCVA, evaluated using a Snellen chart, was converted to logarithm of the minimum angle of resolution (LogMAR) scale.

Statistical analysis was performed to compare the data after treatment with the initial data using the paired $t$-test and to analyse the difference between outcomes in filamentous species and yeast species using the chi-square and Fisher's exact tests with IBM Statistical Package for the Social Sciences (SPSS) Statistics version 24 (SPSS inc., Chicago IL., USA). Quantitative variables were expressed as mean \pm standarddeviation (SD). A p value less than 0.05 was considered statistically significant. Frequencies along with percentages for qualitative variables were used to describe the data.

\section{Results}

A total of 57 patients were identified with fungal keratitis during the study period, of which 14 were excluded due to incomplete records totaling forty-three patients enrolled in this analysis. The mean age was $63.1 \pm 18.6$ years (range 20 to 88 years), most of them above 65 years $(60.5 \%)$. Twenty patients $(46.5 \%)$ were men.

Documented predisposing risk factors were present in 42 of the 43 patients (96.7\%) and are depicted in Table 1. The most common risk factors were use of contact lens (24 eyes, 55.8\%) and long-term use of topical corticosteroids (19 eyes, 44.2\%). Previous keratitis was present in 19 eyes 
Table I Associated Risk Factors for Fungal Keratitis

\begin{tabular}{|l|l|}
\hline Risk Factors & N (\%) \\
\hline Contact Lens & $24(55.8)$ \\
Corticosteroids & $19(44.2)$ \\
Previous keratitis & $19(44.2)$ \\
Previous PK & $14(32.6)$ \\
Ocular surface disease & $12(27.9)$ \\
Traumatic injury with unknown material & $5(11.6)$ \\
Traumatic injury with vegetable matter & $3(7.0)$ \\
\hline Systemic risk factors & \\
Comorbidities & $38(88.4)$ \\
Metabolic syndrome & $21(48.8)$ \\
Diabetes mellitus & $14(32.6)$ \\
\hline
\end{tabular}

Abbreviations: PK, penetrating keratoplasty; \%, percentage.

(44.2\%), of these 4 eyes due to prior HSV keratitis (9.3\%), previous PK was present in 14 eyes (32.6\%) and other ocular surface diseases were present in 12 eyes (27.9\%). Three eyes (7.0\%) suffered recent traumatic corneal injury with vegetable matter and 5 eyes (11.6\%) had previous trauma with an unknown object or material. A total of 38 cases $(88.4 \%)$ had comorbidities of which 14 cases (32.6\%) had diabetes. Metabolic syndrome was presented in 21 cases $(48.8 \%)$.

On the mycological exam, $21(48.8 \%)$ yeast and 22 (51.2\%) filamentous fungi were identify; their respective species are presented in Table 2. Just on one patient two different species were isolated in culture (Candida and Rhodotorula). Slit-lamp examination of four cases and their pathogenic microorganism are identified in Figure 1.

From the onset of symptoms, (sudden ocular pain, photophobia and discharge) to empirical treatment was $12 \pm 15.7$ days. When FK was considered, 19 patients presented hypopyon. The mean of the initial BCVA, documented at hospital admission, was $0.89 \pm 0.67 \log$ MAR. No statistically significant changes were found after treatment ( $\mathrm{p}=0.687$ ) (Table 3).

The medical treatment performed in the studied patients is shown in Table 4 . Nine of the 22 cases ( $40.9 \%$ ) caused by filamentous fungi were treated with voriconazole, 5 $(22.7 \%)$ cases treated with voriconazole and amphotericin B, $3(13.6 \%)$ with clotrimazole and $3(13.6 \%)$ others with fluconazole. Two other cases $(9.1 \%)$ were not treated with any antifungal. In the yeast-like group, 5 cases were treated with amphotericin B (23.8\%), 4 (19.0\%) cases were treated with clotrimazole, 3 (14.3\%) with voriconazole, 1 (4.8\%) with voriconazole and amphotericin B and $5(23.8 \%)$ with
Table 2 Pathogenic Organisms Identified in Cases of Fungal Keratitis

\begin{tabular}{|l|l|}
\hline Organism & N (\%) \\
\hline Yeast species & 2 I (48.8) \\
Candida parapsilosis & I I (25.6) \\
Candida albicans & $7(16.3)$ \\
Candida glabrata & $\mathrm{I}(2.3)$ \\
Candida famata & $\mathrm{I}(2.3)$ \\
Rhodotorula mucilaginosa & $\mathrm{I}(2.3)$ \\
\hline Filamentous species & $22(5 \mathrm{I} .2)$ \\
Fusarium spp. & $\mathrm{I} 0(23.4)$ \\
Aspergillus fumigatus & $2(4.7)$ \\
Aspergillus niger & $\mathrm{I}(2.3)$ \\
Aspergillus flavus & $\mathrm{I}(2.3)$ \\
Paecilomyces spp. & $3(7.0)$ \\
Mucor spp. & $\mathrm{I}(2.3)$ \\
Scedosporium spp. & $\mathrm{I}(2.3)$ \\
Beauveria spp. & $\mathrm{I}(2.3)$ \\
Acremonium spp. & $\mathrm{I}(2.3)$ \\
Alternaria spp. & $\mathrm{Iv}(2.3)$ \\
\hline
\end{tabular}

fluconazole. Three of the $21(14.3 \%)$ were not treated with any eyedrop.

Surgical intervention was required, in 24 (55.8\%) patients. Twenty-three eyes (53.5\%) underwent therapeutic PK, of which 12 due to corneal perforation. Seventeen of the 23 patients $(73.9 \%)$ that underwent PK, were also submitted to anterior chamber injection of antifungal drugs [fifteen $(88.2 \%)$ with voriconazole, 1 (5.88\%) with amphotericin and another with an antibiotic (5.88\%)]. The averaged time between antifungal drug prescription and penetrating keratoplasty was $27.6 \pm 20.3$ days. Nine of the 14 eyes (64.3\%) with prior PK required surgical interventions, of which 8 were repeated PKs. Over the follow-up period, 5 patients developed endophthalmitis, one developed phthisis bulbi and evisceration was the outcome in 12 patients.

When we compared the clinical profiles in yeast and filamentous cases (Table 5), yeast had a statistically significant higher prevalence in long-term users of topical corticosteroids compared to filamentous ( $p=0.043$ ). There was also a tendency for increased need of PK in keratitis by filamentous fungi $(p=0.082)$. There were no other statistically significant differences at the studied variables such as previous ocular trauma with vegetable matter, previous trauma with unknown object or material, contact lens, previous PK, other ocular surface diseases, previous keratitis, diabetes mellitus, comorbidities, phthisis bulbi and endophthalmitis $(\mathrm{p}=0.482, \mathrm{p}=0.158, \mathrm{p}=$ 


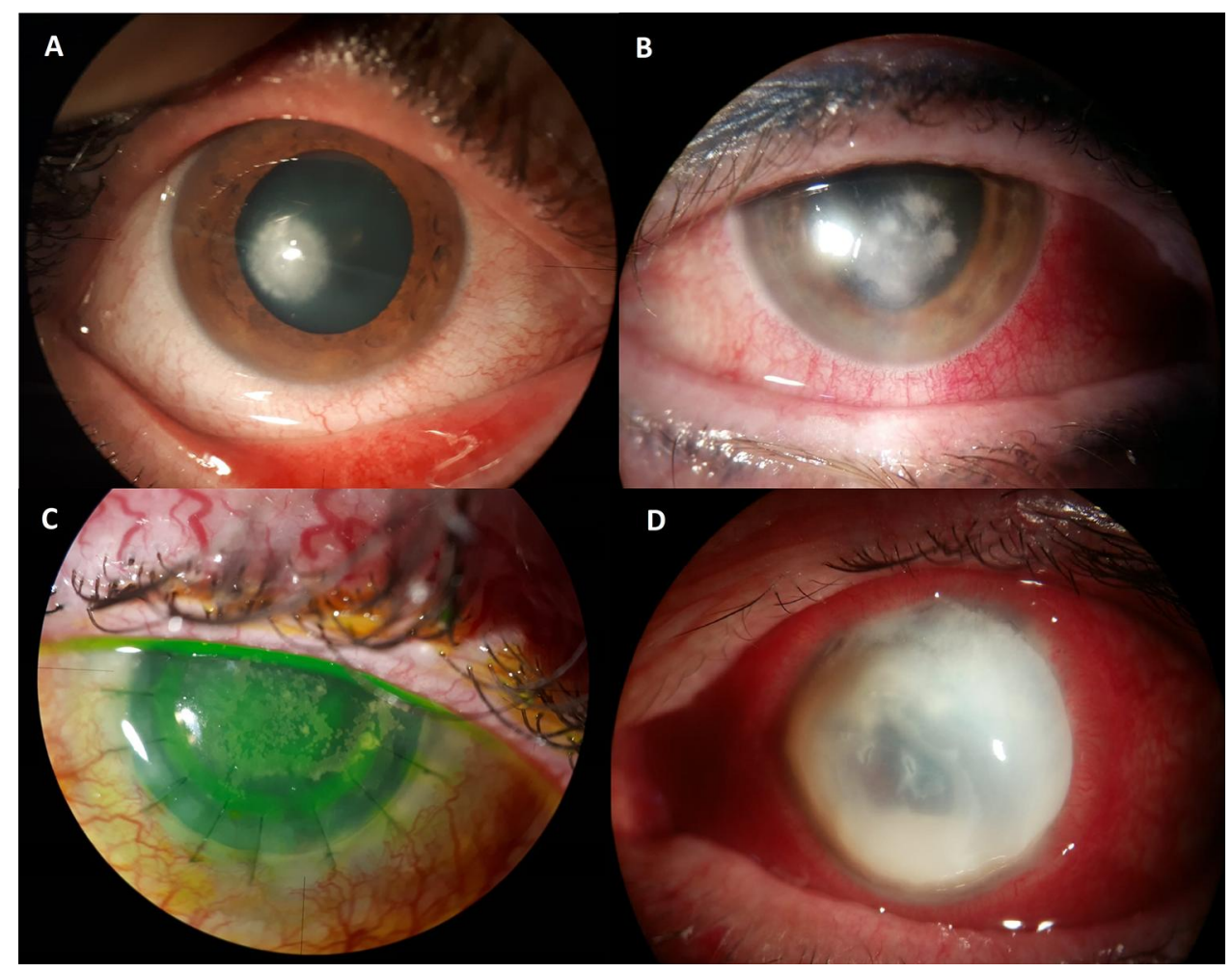

Figure I Slit-lamp examination of four cases of fungal keratitis ((A) Beauveria bassiana; (B) Fusarium; (C) Candida albicans; (D) Fusarium).

$0.475, \mathrm{p}=0.192, \mathrm{p}=0.332, \mathrm{p}=0.578, \mathrm{p}=0.414, \mathrm{p}=$ $0.477, \mathrm{p}=0.488, \mathrm{p}=0.477$, respectively).

Both groups presented similar BCVA at presentation and at the end of follow-up ( $p=0.997, p=0.169$, respectively; Table 6).

Regarding evisceration, 12 eyes (27.9\%) underwent this procedure. No differences were found in evisceration development between those who did and did not undergo PK $(p=0.265)$. No association between evisceration and the type of fungi involved was detected $(p=0.332)$.

\section{Discussion}

Fungal keratitis is an important cause of infectious keratitis and ocular morbidity. Studies have shown several risk

Table 3 Mean Changes in Visual Between Presentation and After Treatment

\begin{tabular}{|l|l|l|l|}
\hline Variables & Initial & After Treatment & \multirow{2}{*}{ P value } \\
\cline { 2 - 3 } & $\mathbf{N}=\mathbf{2 4}$ & $\mathbf{N}=\mathbf{2 6}$ & \\
\cline { 2 - 3 } & Mean \pm SD & Mean \pm SD & \\
\hline BCVA (logMar) & $0.89 \pm 0.67$ & $1.30 \pm 0.80$ & 0.687 \\
\hline
\end{tabular}

Abbreviations: BCVA, best corrected visual acuity; logMAR, logarithm of minimal angle of resolution; SD, standard deviation. factors related to this infectious corneal disease. In our study, contact lens (55.8\%), long term use of topical corticosteroids (44.2\%), previous keratitis (44.2\%) and previous PK (32.6\%) were the leading risk factors and are in agreement with those found in reports of FK in other European countries. ${ }^{4}$

The use of contact lens, the major risk factor found in our research contributes to FK by several mechanisms, such as interruption of normal tear flow (an essential component of corneal immunity), corneal epithelium

Table 4 Antifungals Used in the Treatment of Yeast-Like and Filamentous Fungi

\begin{tabular}{|l|l|l|}
\hline Variables & $\begin{array}{l}\text { Yeast-Like } \\
\text { Fungi }\end{array}$ & $\begin{array}{l}\text { Filamentous } \\
\text { Fungi }\end{array}$ \\
\cline { 2 - 3 } & $\mathbf{N = 2 \text { I }}$ & $\mathbf{N = 2 2}$ \\
\cline { 2 - 3 } & $\mathbf{N}(\%)$ & $\mathbf{N}(\%)$ \\
\hline $\begin{array}{l}\text { Eyedrops } \\
\text { Voriconazole }\end{array}$ & $3(14.3)$ & $9(40.9)$ \\
Amphotericin B & $5(23.8)$ & 0 \\
Voriconazole + Amphotericin B & $1(4.8)$ & $5(22.7)$ \\
Clotrimazole & $4(19.0)$ & $3(13.6)$ \\
Fluconazole & $5(23.8)$ & $3(13.6)$ \\
\hline
\end{tabular}

Abbreviation: \%, percentage. 
Table 5 Clinical Profiles Compared in Yeast-Like and Filamentous Cases

\begin{tabular}{|c|c|c|c|}
\hline \multirow[t]{3}{*}{ Variables } & $\begin{array}{l}\text { Yeast-Like } \\
\text { Fungi }\end{array}$ & $\begin{array}{l}\text { Filamentous } \\
\text { Fungi }\end{array}$ & \multirow[t]{3}{*}{$P$ value } \\
\hline & $\mathbf{N}=\mathbf{2} \mathbf{I}$ & $\mathbf{N}=\mathbf{2 2}$ & \\
\hline & $\mathbf{N}(\%)$ & $\mathbf{N}(\%)$ & \\
\hline \multicolumn{4}{|l|}{ Risk factors } \\
\hline Corticosteroids & $13(62.0)$ & $6(27.3)$ & 0.043 \\
\hline Plants trauma & I (4.8) & $2(9.1)$ & 0.482 \\
\hline Trauma (other causes) & I (4.8) & $4(18.2)$ & 0.158 \\
\hline Previous PK & $9(42.9)$ & $5(22.7)$ & 0.192 \\
\hline Contact Lens & $12(57.1)$ & $12(54.5)$ & 0.475 \\
\hline Previous Keratitis & $8(38.1)$ & $8(36.4)$ & 0.578 \\
\hline Ocular surface disease & $7(33.3)$ & $5(22.7)$ & 0.332 \\
\hline Comorbidities & I8 (85.7) & $18(81.8)$ & 0.477 \\
\hline Diabetes mellitus & $8(38.1)$ & $6(27.3)$ & 0.414 \\
\hline Metabolic Syndrome & II (52.4) & $10(45.5)$ & 0.559 \\
\hline \multicolumn{4}{|l|}{ Treatment } \\
\hline Therapeutic PK & $9(42.9)$ & $14(63.6)$ & 0.082 \\
\hline Anterior Chamber & & & \\
\hline \multicolumn{4}{|l|}{ Injection } \\
\hline Voriconazole & $5(23.8)$ & $10(45.5)$ & \\
\hline Amphotericin B & I (4.8) & 0 & 0.170 \\
\hline Antibiotic & 0 & I (4.5) & \\
\hline \multicolumn{4}{|l|}{ Clinical Outcomes } \\
\hline Phthisis bulbi & 0 & I (4.5) & 0.488 \\
\hline Endophthalmitis & $2(9.5)$ & $3(13.6)$ & 0.477 \\
\hline Evisceration & $5(23.8)$ & $7(31.8)$ & 0.332 \\
\hline
\end{tabular}

Abbreviations: PK, penetrating keratoplasty; \%, percentage.

microtrauma, changes in ocular surface immunity and corneal hypoxia. Equally, the prior topical corticosteroids use, the second risk factor observed in our study, could be justified by steroid capacity to promote fungal proliferation. Regarding previous PK, this risk factor was present in $32.6 \%$ of our cases. Our study presents a higher incidence than previous published data of $8.9 \%{ }^{9}$ to $24 \%{ }^{8}$

Moreover, some systemic factors can contribute to increased risk of fungal infection. Diabetic patients show more severe clinical manifestations and worse prognosis in some reports. Diabetes was present in $32.6 \%$ of our studied patients, a higher percentage than other reports ( $7.1 \%$ to $16 \%),{ }^{8,9}$ which can be related with the older age of our sample $(60.5 \%$ were older than 65 years of age).

The highest prevalence of fungal keratitis is usually described in hot and humid climate zones, where it may constitute $30-62 \%$ of all cases of keratitis. ${ }^{10,11}$ However, in recent years, the prevalence of fungal keratitis in moderate climate zones, like Europe, has been increasing. ${ }^{12}$ The types and distribution of the microbiological profile of fungal keratitis vary according to geography, climate and the socioeconomic characteristics of the involved patients. In our study, filamentous and yeast species have equally prevalent aetiologist (51.2\% versus $48.8 \%$, respectively). Candida species $(46.5 \%)$, the most common fungi identified in our series, were followed by Fusarium species (23.4\%). Our findingin relation to the Candida species is like to the results of other studies conducted across the globe (Pennsylvania (45.8\%), ${ }^{13}$ Denmark (52\%) ${ }^{14}$ Paris $(58 \%)^{15}$ and London $\left.(60.6 \%)\right) .{ }^{16}$

Regarding the patients' age at the time of diagnosis, a mean of 63.1 years old was noticed. When we analyse the mean age according to the microbiological profile, we found a not significant higher mean age for yeast species (65.8 years old) than for filamentous species (60.3 years old). This difference was more pronounced in other European studies. ${ }^{4}$

Other differences between yeast and filamentous have been described. Yeasts seems to have higher prevalence in patients with known ocular surface disorders, in those after previous ocular surgery and in long-term users of topical corticosteroids while filamentous are most often isolated from keratitis associated with ocular trauma and contact lens wear. ${ }^{4}$ In our study, we also find that yeast had a statistically significant higher prevalence in long-term users of topical corticosteroids compared to filamentous species. None of the other previously mentioned risk factors were different between yeast and filamentous groups.

The overall surgical intervention (anterior chamber injection with antifungal and/or PK) rate found, in our study, was $55.8 \%$ and from these patients only one did not undergo

Table 6 Visual Outcomes in Filamentous and Yeast-Like Cases of Fungal Keratitis

\begin{tabular}{|l|l|l|l|}
\hline \multirow{2}{*}{ Variables } & Yeast-Like Fungi & Filamentous Fungi \\
\cline { 2 - 3 } & $\mathbf{N}=\mathbf{2 1}$ & $\mathbf{N}=\mathbf{2 2}$ & \\
\cline { 2 - 4 } & Mean \pm SD & Mean \pm SD & 0.997 \\
\hline Initial BCVA (logMar) & $0.128 \pm 0.232$ & $0.129 \pm 0.203$ & 0.169 \\
\hline BCVA after treatment (logMar) & $0.005 \pm 0.005$ & $0.089 \pm 0.214$ & \\
\hline
\end{tabular}

Abbreviations: BCVA, best corrected visual acuity; logMAR, logarithm of minimal angle of resolution; SD, standard deviation. 
therapeutic PK. We also described a tendency for increased need of PK in keratitis by filamentous fungi, like in similar studies. ${ }^{4,8}$ The percentage of patients receiving corneal grafts performed in different countries, varies from $24 \%$ to $52 \%{ }^{14-16}$ Our keratoplasty rate $(53.5 \%)$ was similar to that described by Galarreta et al in London. ${ }^{16}$ Eyes with fungal keratitis were reported to have an evisceration or enucleation rate of $4.26-12 \%$. $^{2,17}$ The evisceration rate in the current study was higher (27.9\%, 12 of 43 eyes). When studies evaluated eyes with endophthalmitis caused by infectious keratitis the evisceration rate increased from $31 \%$ to $62.2 \%{ }^{18}$

This study also has some limitations. It provides a retrospective review of clinical data from a single centre. A small number of patients were included in this study, even though records covered a period of 10 years. Only patients with microbiological evidence of fungal keratitis were enrolled in this study while cases without such evidence were excluded, even if fungal keratitis was highly suspected. Despite such limitations, this study has an important clinical significance, as it reflects the complexity behind fungal keratitis. A lot of factors could explain those difficulties, such as late clinical presentation, uncertain timing on antifungal treatment and antifungal resistance spectrum.

There is a need to continue research on aetiology and diagnostic and therapeutic management of fungal keratitis.

\section{Conclusion}

In conclusion, our results mirror how difficult and challenging the approach and treatment of fungal keratitis can be. Therefore, similar studies should be performed to formulate better diagnostic approaches and treatment strategies, and to improve a patient's outcome.

\section{Funding}

None of the authors have received grants for this submission.

\section{Disclosure}

The authors report no conflicts of interest for this work.

\section{References}

1. Ansari Z, Miller D, Galor A. Current thoughts in fungal keratitis: diagnosis and treatment. Curr Fungal Infect. 2013;7:209-218. doi:10.1007/s12281-013-0150-1
2. Mohd-Tahir F, Norhayati A, Siti-Raihan I, Ibrahim M. A 5-year retrospective review of fungal keratitis at hospital universiti sains malaysia. Interdiscip Perspect Infect Dis. 2012;2012:851563. doi:10.1155/2012/851563

3. Mahmoudi S, Masoomi A, Ahmadikia K, et al. Fungal keratitis: an overview of clinical and laboratory aspects. Mycoses. 2018;61:916-930. doi:10.1111/myc.12822

4. Nowik KE, Wylęgała A, Nowik K, et al. A single-centre retrospective observational study of fungal keratitis in Poland with a review of findings in Europe. Ann Agric Environ Med. 2020;27(3):343-347. doi: $10.26444 / \mathrm{aaem} / 109414$

5. Wu J, Zhang WS, Zhao J, Zhou HY. Review of clinical and basic approaches of fungal keratitis. Int J Ophthalmol. 2016;9:1676-1683.

6. Manikandan P, Abdel-Hadi A, Randhir Babu Singh Y, et al. Fungal keratitis: epidemiology, rapid detection, and antifungal susceptibilities of Fusarium and Aspergillus isolates from corneal scrapings. Biomed Res Int. 2019;2019:6395840. doi:10.1155/2019/6395840

7. Day S, Lalitha P, Haug S, et al. Activity of antibiotics against Fusarium and Aspergillus. Br J Ophthalmol. 2009;91(1):116-119. doi:10.1136/bjo.2008.142364

8. Ho JW, Fernandez MM, Rebong RA. Microbiological profiles of fungal keratitis: a 10-year study at a tertiary referral center. J Ophthalmic Inflamm Infect. 2016;6(1):5. doi:10.1186/s12348-0160071-6

9. Iyer SA, Tuli SS, Wagoner RC. Fungal keratitis: emerging trends and treatment outcomes. Eye Contact Lens. 2006;32(6):267-271. doi:10.1097/01.icl.0000249595.27520.2e

10. Chang HY, Chodosh J. Diagnostic and therapeutic considerations in fungal keratitis. Int Ophthalmol Clin. 2011;51:33-42. doi:10.1097/ IIO.0b013e31822d64dc

11. Gopinathan U, Sharma S, Garg P, Rao GN. Review of epidemiological features, microbiological diagnosis and treatment outcome of microbial keratitis: experience of over a decade. Indian J Ophthalmol. 2009;57:273-279. doi:10.4103/0301-4738.53051

12. Walkden A, Fullwood C, Tan SZ, et al. Association between season, temperature and causative organism in microbial keratitis in the UK. Cornea. 2018;37(12):1555-1560. doi:10.1097/ICO.0000000000 001748

13. Tanure MA, Cohen EJ, Sudesh S, Rapuano CJ, Laibson PR. Spectrum of fungal keratitis at wills eye hospital, Philadelphia, Pennsylvania. Cornea. 2000;19(3):307-312. doi:10.1097/00003226200005000-00010

14. Nielsen SE, Nielsen E, Julian HO, et al. Incidence and clinical characteristics of fungal keratitis in a Danish population from 2000 to 2013. Acta Ophthalmol. 2015;93(1):54-58. doi:10.1111/aos.12440

15. Rondeau N, Bourcier T, Chaumeil C, et al. Fungal keratitis at the Centre Hospitalier national d'Ophtalmologie des Quinze-Vingts: retrospective study of 19 cases. J Fr Ophtalmol. 2002;25(9):890-896.

16. Galarreta DJ, Tuft SJ, Ramsay A, Dart JK. Fungal keratitis in London: microbiological and clinical evaluation. Cornea. 2007;26 (9):1082-1086. doi:10.1097/ICO.0b013e318142bff3

17. Iselin KC, Baenninger PB, Schmittinger-Zirm A, Thiel MA, Kaufmann C. Fungal keratitis: a six-year review at a tertiary referral centre. Klin Monbl Augenheilkd. 2017;234(4):419-425. doi:10.1055/ s-0042-123233

18. Farrell S, McElnea E, Moran S, Knowles S, Murphy CC. Fungal keratitis in the Republic of Ireland. Eye (Lond). 2017;31 (10):1427-1434. doi:10.1038/eye.2017.82 


\section{Publish your work in this journal}

Clinical Ophthalmology is an international, peer-reviewed journal covering all subspecialties within ophthalmology. Key topics include: Optometry; Visual science; Pharmacology and drug therapy in eye diseases; Basic Sciences; Primary and Secondary eye care; Patient Safety and Quality of Care Improvements. This journal is indexed on PubMed
Central and CAS, and is the official journal of The Society of Clinical Ophthalmology (SCO). The manuscript management system is completely online and includes a very quick and fair peer-review system, which is all easy to use. Visit http://www.dovepress.com/ testimonials.php to read real quotes from published authors. 